\title{
Towards self-clocked gated OCDMA receiver
}

S. Idris

Siti.idris-othman@strath.ac.uk

\section{T. Osadola}

\section{Glesk}

\begin{abstract}
Department of Electrical and Electronic Engineering, University of Strathclyde, Royal College Building, 204 George Street, Glasgow G1 1XW, United Kingdom

Department of Electrical and Electronic Engineering, University of Strathclyde, Royal College Building, 204 George Street, Glasgow G1 1XW, United Kingdom

Department of Electrical and Electronic Engineering, University of Strathclyde, Royal College Building, 204 George Street, Glasgow G1 1XW, United Kingdom
\end{abstract}

A novel incoherent OCDMA receiver with incorporated all-optical clock recovery for self-synchronization of a time gate for the multi access interferences (MAI) suppression and minimizing the effect of data time jitter in incoherent OCDMA system was successfully developed and demonstrated. The solution was implemented and tested in a multiuser environment in an out of the laboratory OCDMA testbed with two-dimensional wavelength-hopping time-spreading coding scheme and $0 \mathrm{C}-48$ ( $2.5 \mathrm{Gbp} / \mathrm{s})$ data rate. The self-clocked all-optical time gate uses SOA-based fibre ring laser optical clock, recovered all-optically from the received OCDMA traffic to control its switching window for cleaning the autocorrelation peak from the surrounding MAI. A wider eye opening was achieved when the all-optically recovered clock from received data was used for synchronization if compared to a static approach with the RF clock being generated by a RF synthesizer. Clean eye diagram was also achieved when recovered clock is used to drive time gating.

[DOI: http://dx.doi.org/10.2971/jeos.2013.13013]

Keywords: All-optical clock recovery (AOCR), incoherent optical code division multiple access (OCDMA), SOA-based MZI all-optical time gate, two-dimensional wavelength- hopping time-spreading code, self-clocking

\section{INTRODUCTION}

Optical code division multiple access (OCDMA) is a multiple access technique where signal is transmitted in the form of unique codes. It possess some advantages over other techniques in terms of flexibility in data rates, provides enhanced capacity and quality of service depending on the network demands, and support for a bursty traffic [1, 2]. On the other hand, OCDMA performance is primarily influenced by the multi-access interference (MAI). This is also the case for two-dimensional wavelength-hopping time-spreading (2DWHTS) OCDMA codes and is a result of a nonzero cross correlation among codes used by simultaneous users [1]. The use of 2D-WHTS family of codes has been widely researched for possible use in different future OCDMA applications [3]. The codes are characterized by the combination of time spreading and wavelength hopping of picosecond pulse patterns. This coding approach spreads the pulses in both the time and wavelength domain simultaneously thus achieving code flexibility as well as performance $[4,5]$. This approach has several advantages including reduced cross correlation, increased cardinality and nonexistence of autocorrelation side lobes [6]. Widely studied for both time spreading and wavelength hopping has been the family of prime codes [2], [6] employing multi-wavelength short pulses for codes generation. The WHTS receiver receives signals from all users on the network. The decoder discriminates between intended and interfering data streams by correlating the received signal with the appropriate code matrix. The decoder thus undoes the time spreading for the appropriate code sequence and aligns its wavelength pulses back in time. When the matched code sequence is received, output of the decoder consists of few pulses which are aligns on top of each other known as an autocorrelation peak. For the other unmatched code sequences to the decoder, the code sequence is further spread over the bit period resulting in lower intensity MAI. MAI increases with the growing number of simultaneous users resulting in deterioration in the bit error rate (BER) and system performance $[1,2]$. Eventually, it upper limits the maximum number of simultaneous users at the given BER, the property known as the soft blocking. Analyses have shown that MAI is still the dominant effect even when an optical beat noise among optical pulses of the same wavelength from different users is taken into account [7]. Research efforts to reduce MAI in OCDMA systems have taken many directions, including development of novel coding schemes as well as hardware techniques that either help to reduce MAI or provide improved performance in the presence of MAI.

Besides detrimental effects of MAI, the transmitting signal especially at high data rates over longer distances often suffers from timing jitter which leads to receiver synchronization issues resulting in BER degradation. Accurate and "dynamic" synchronization of the receiver is needed to improve OCDMA signal detection to ensure that the OCDMA receiver is as little as possible affected by the timing jitter. A clock recovery 
for the receiver synchronization is a well-known approach for addressing this issue and clock recovery subsystems have been recognized as essential for high speed detection systems [2], [7, 8]. Implementations of wide variety of clock extraction techniques [8] for use in receiver synchronization were predominantly developed for wavelength division multiplexing (WDM) $[9,10]$ and optical time division multiplexing (OTDM) $[11,12]$ systems. However, their application in OCDMA using picosecond pulses is limited or often impossible. Clock and data recovery techniques for such OCDMA systems were reported in $[13,14]$. However, these approaches were not implemented all-optically. Clock recovery approach for suppressing the effect of jitter in an OCDMA signal was demonstrated by using a nonlinear optical loop mirror (NOLM) or terahertz optical asymmetric multiplexer (TOAD) $[15,16]$. However, a realization of suitable all-optical clock extraction circuit which will recover clock signal from the incoming OCDMA data stream and can produce optical clock without any intermediate electronic stage is still needed. In principle, recovering an optical clock from incoming data at a given bit rate means extracting a periodic signal with period reciprocal of the bit rate, while keeping it free of information carried by data and without a phase noise [2].

Several techniques have been also proposed for MAI reduction for use in OCDMA systems. Some examples are: optical "time gating" and "optical thresholding" of the recovered autocorrelation peak. In their comparison to encoding and balanced detection techniques, these techniques have the advantage of being compatible with wider family of codes and are also suitable for ultra-high data rates [2]. However, for implementation of the optical time gating technique an optical clock is needed to precisely control the position of the switching window of the time gate for allowing to pass the user decoded OCDMA data referred to as autocorrelation peak [17] while blocking the MAI known as cross-correlations that falls outside of it (see Figure 1). In contrast to the time gating, as already discussed, the optical thresholder can be used to select the autocorrelation - the highest intensity peak for passing while rejecting the MAI - the lower intensity peaks [2]. However, when "cleaning" the autocorrelation we need to keep in mind that systems used for reduction of MAI in the time domain have their limitations such as a finite extinction ratio of sampling gates and a crosstalk produced by MAI pulses in adjacent chips to autocorrelation peak [18]. In the wavelength domain, crosstalk from adjacent wavelength channels can also lead to spreading of the energy over the bit period in the time domain.

Various encoding and decoding methods exploring different technologies and approaches exists and were demonstrated for use with incoherent two dimensional OCDMA encoders and decoders. These include the use of thin film filters in combination with optical delay lines (ODL) [19], holographic Bragg gratings [20], and fibre Bragg gratings (FBG) [21]. Of the above mentioned methods, it is worthy of noting that FBG while inexpensive, provide very promising approach towards miniaturisation and complexity as the function of wavelength filtering is combined with desired time delays in one single fibre device [22]. FBGs have been widely used in optical communications signal processing for some time [21]. The use of

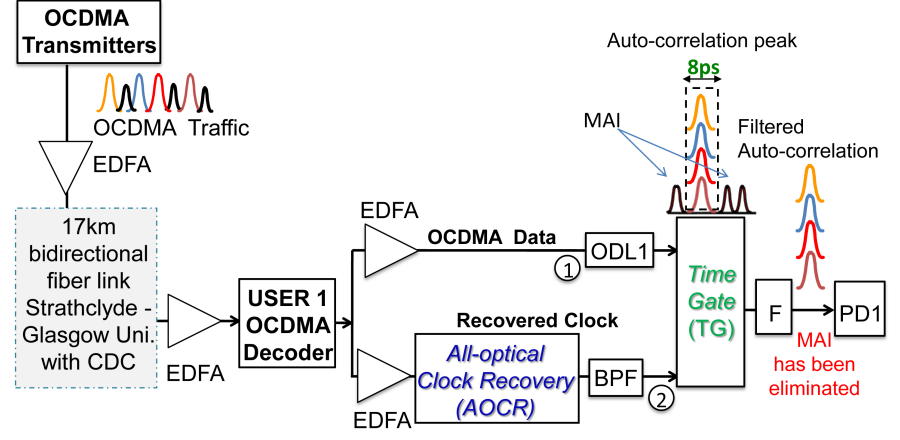

FIG. 1 The concept of OCDMA optical time gating with the need for AOCR for synchronization and to open switching window for MAI elimination where ODL1 is an optical delay line, BPF - band pass filter, PD - photo-detector, F - low pass filter, CDC chromatic dispersion compensation with picoseconds accuracy.

FBGs for encoding and decoding of WHTS optical codes in OCDMA systems by spreading multiple wavelengths within a bit period and placing them into time chips at the transmitter end and reciprocally de-spreading them and forming an autocorrelation peak at the receiver end is a widely known technique $[21,22]$.

In this paper we report for the first time a demonstration of an incoherent OCDMA receiver with the self-clocked time gate for MAI reduction. The receiver has incorporated all-optical clock recovery (AOCR) subsystem based on the built-in semiconductor optical amplifier (SOA) based fibre ring laser. The receiver was tested in a $17 \mathrm{~km}$ long OCDMA out of the laboratory testbed which has been compensated for chromatic dispersion with a sub-picosecond accuracy. The quality of the all-optically recovered optical clock was also evaluated for its suitability to drive the built-in all-optical picosecond time gate, i.e., a semiconductor optical amplifier - based $2 \times 2$ Mach-Zehnder Interferometric Switch, (SOA-MZIS) used in the OCDMA receiver to filter out MAI noise from the received multiuser signal.

\section{SELF CLOCKED TIME GATE FOR MAI ELIMINATION / REDUCTION}

The concept of MAI elimination by a self-clocked optical time gate we used is depicted in Figure 1. The OCDMA traffic generated by OCDMA transmitters sent through $17 \mathrm{~km}$ long bidirectional fibre link is first decoded by the User 1 OCDMA decoder. The outcome is an autocorrelation peak (User 1 OCDMA data) and the cross correlation known as MAI. The decoder output is then divided into two signals, both amplified by using EDFAs. The signal of the upper branch is sampled by the all-optical time gate (TG) in order to filter out the MAI [23, 24]. Here, an optical delay line (ODL1) is used to set proper timing between the gating window generated by the TG and the incoming autocorrelation peak. The signal in the lower branch is used to obtain optical clock which is then used to control the TG operation. A band pass filter (BPF) is used after the EDFA to pass autocorrelation peak and remove the amplified spontaneous emission, ASE. By reducing both, the MAI and timing jitter, power budget improvements can be expected which will be subject of our further investigation. 


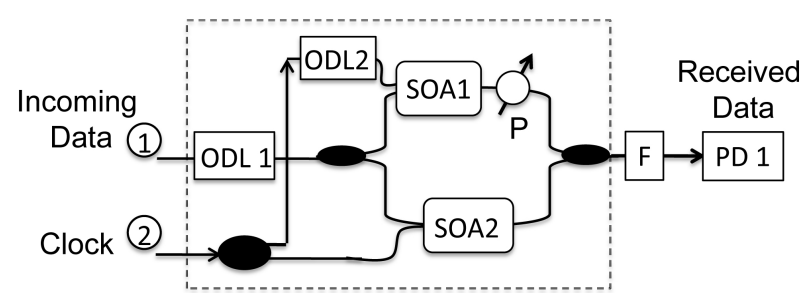

FIC. 2 SOA-MZI switch. SOA1 and SOA2 are Semiconductor Optical Amplifiers, ODLoptical delay line, F - low pass filter, P - phase adjustment heating element.

\subsection{SOA-based MZI switch as the Time Gating (TG)}

The implementation of all-optical picosecond time gating requires precise TG control by using optical clock to open and close the switching windows of the TG. All-optical ultrafast switches are ideal devices for this type of optical signal processing. The continuing research efforts in photonic integration have led to the development of a high speed SOA-based all-optical switching devices [25] with a good potential in the variety of signal processing applications [26]. The semiconductor optical amplifier based Mach-Zehnder interferometer (SOA-MZI) we used is an integrated all-optical switch which can support ultrafast switching applications [27]. Conceptually, the device operation is well known and quite straightforward. The switching is achieved by inducing differential phase shifts by SOAs, each located in one arm of the MZ interferometer [25]. The carrier dynamics enables this switch to be suitable for all-optical signal processing at ultra-high speeds [28].

Figure 2 is a schematic diagram of the integrated SOA-MZI switch we used in our experiment (40G-2R2-ORP from CIP Photonics). Here, in order to create the desired switching window to match the autocorrelation width of $8 \mathrm{ps}$ a differential delay needed between both SOA-MZI control signals was set by using an optical delay line (ODL2) after splitting the all-optically recovered clock by AOCR (see Figure 1). At the switch output optical low pass filter, $\mathrm{F}$ is deployed to block the passing clock and pass the received OCDMA data cleared of MAI before entering a photo detector PD (Nortel $10 \mathrm{~Gb} / \mathrm{s}$ NRZ receiver with $-18 \mathrm{dBm}$ sensitivity) (see Figures 1 and 2).

\subsection{All-optical clock recovery (AOCR) from oCDMA signal}

Any transmission system transmitting data at longer distances will potentially suffer from the detrimental effects of timing jitter. Due to time jitter, an all-optical clock recovery is essential especially if the OCDMA receiver uses an all-optical time gate for all-optical post processing (with picosecond accuracy) of the received OCDMA data to remove MAI. It is therefore essential to recover its synchronization signal alloptically from the incoming data in order to eliminate the effect of jitter to enhance data detection. Figure 3 is a schematic diagram of our approach we developed for all-optical clock recovery from the incoming OCDMA traffic for use in incoherent OCDMA system [29].

The incoming traffic is first decoded by OCDMA decoder to
OCDMA Receiver with AOCR

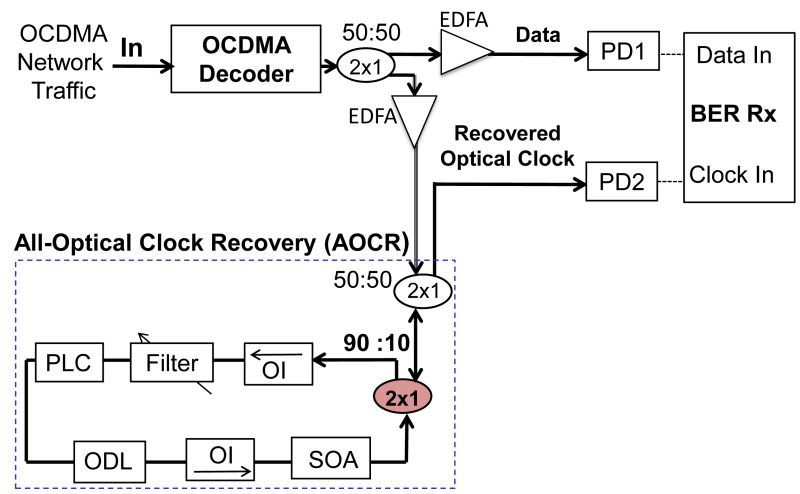

FIG. 3 OCDMA Receiver with built-in all-optical clock recovery where: SOA is Semiconductor Optical Amplifier, OI - Optical Isolator, ODL- Optical Delay Line, PLC- Polarization Controller, PD- photo-detector, BER Rx - bit error rate receiver.

produce OCDMA autocorrelation peak which is then split into two ways: one is used for OCDMA data detection while the other is sent to the AOCR circuit to produce the optical clock at the repetition rate of the incoming OCDMA data.

The SOA based fibre ring laser AOCR operation relies on the fast gain saturation of the SOA by the incoming OCDMA data stream resulting in the laser cavity modulation which under right conditions will lead to the fiber ring laser locking. The cavity of the AOCR was constructed from fibre pigtailed devices as indicated in Figure 3. The incoming data pattern and recovered optical clock are introduced into / extracted out of the loop, respectively by a $2 \times 1$ optical fibre coupler with the power splitting ratio $(100-x) / x$. Two different coupling configurations (with $x=10$ and $x=20$ ) were tested to look for optimized performance of the AOCR. Better results were obtained using $\mathrm{x}=10$. A $5 \mathrm{~nm}$ tuneable optical filter inside the loop was used for the wavelength selection for the recovered optical clock and a variable optical delay line (ODL) for adjusting the cavity length of the AOCR circuit for precise matching of the data rate (OC-48) of the incoming signal. When the ODL is adjusted so that the repetition rate of the OCDMA input signal is equal to an integer multiples of the fundamental frequency of the fibre ring cavity, the AOCR circuit becomes properly synchronized with the input signal the cavity becomes harmonically locked and optical clock signal is generated. Two optical isolators inside the loop maintain the direction of the signal circulation. The all-optically generated clock by the AOCR was then evaluated by using BER tester for its stability and later for its suitability to be used as the optical control to drive the SOA-MZI all-optical switch (TG).

\section{EXPERIMENTAL RESULT}

The setup shown in Figure 4 is a layout of the incoherent OCDMA field-based testbed we used for the receiver testing. It consists of a multiuser OCDMA transmitter (comprising four simultaneously transmitting OCDMA users), a $17 \mathrm{~km}$ long bidirectional fibre optic link with sub picosecond chromatic dispersion compensation (CDC), and the receiver 


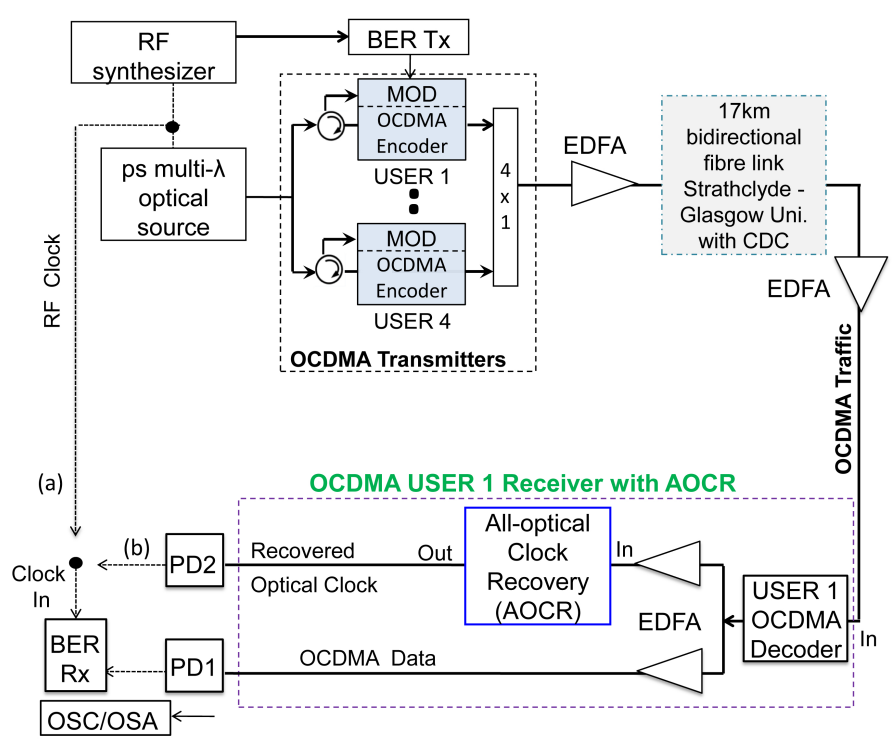

FIG. 4 Field based multi user OCDMA test bed with OCDMA User 1 receiver under the test under two scenarios:

(a) BER Rx is synchronized directly from an RF synthesizer

(b) BER Rx is synchronized by the all-optically recovered clock generated by the OCDMA receiver with built-in $A O C R$.

AOCR - all-optical clock recovery, BER Tx/Rx - bit error rate Transmitter/ Receiver, MOD -LiNb03 Mach-Zehnder data modulator, RF - radio frequency, PD - photo detector, OSC - bandwidth limited digitizing oscilloscope with optical sampling head, OSA optical spectrum analyser, CDC - chromatic dispersion compensation with a picosecond accuracy.

under the test. The system uses incoherent two-dimensional wavelength-hopping time-spreading (2D-WHTS) OCDMA codes [1]. A picosecond multi wavelength source is used to generate four wavelengths pulses ( $\sim 5$ ps FWHM) at $\lambda_{1}=1551.72 \mathrm{~nm}, \lambda_{2}=1550.92 \mathrm{~nm}, \lambda_{3}=1552.52 \mathrm{~nm}$, and $\lambda_{4}=1550.12 \mathrm{~nm}$ which are placed by OCDMA encoders based on fibre Bragg gratings (FBG) [21] within 53 time chips, each having duration of $\sim 8$ ps, thus creating so called $(4,53)$ 2D-WHTS family of OCDMA codes [2]. The system running at OC-48 uses $2^{7}-1$ PRBS pattern generated by an Agilent N4903A BER tester to generate transmitted data.

On a receiving site, Figure 4 shows the device under the test - the newly developed incoherent OCDMA receiver with a built-in AOCR, here as being used by the User 1 . The receiver after decoding the traffic generated by four OCDMA simultaneous users outputs OCDMA User 1 data represented by the autocorrelation peak as well as the all-optically recovered clock signal (see Figure 3 for more details).

\subsection{AOCR evaluation}

To evaluate the AOCR performance, the BER measurements were recorded for received data by the User 1 taken under two different scenarios of synchronization (see Figure 4):

(a) Using the clock signal generated localy by the RF synthesizer (Agilent E4432B);

(b) Using the all-optically recovered clock by the receiver. Optical spectrum analyzer (Agilent 86146B) and bandwidth limited digitizing oscilloscope with a $20 \mathrm{GHz}$ optical sampling (a) Eye diagram of $2^{7}-1$ PRBS data

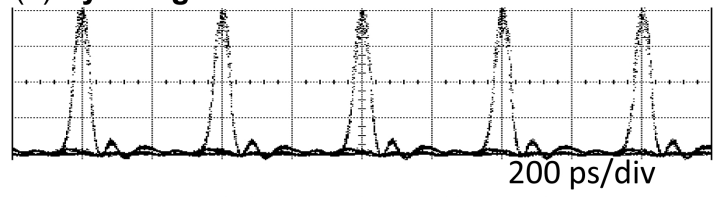

(b) Example of data pattern of ..1010..

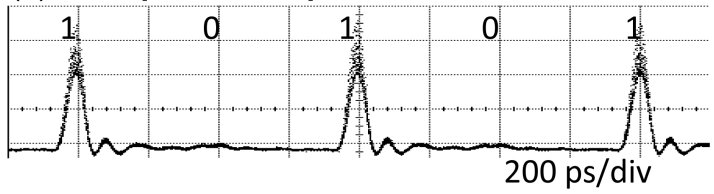

(c) All-optically recovered clock signal

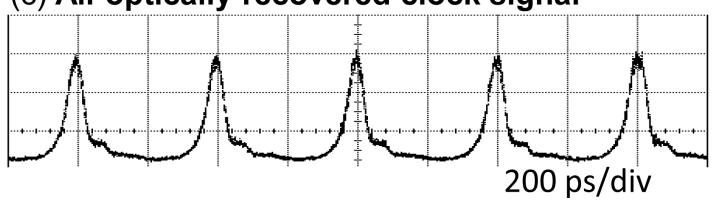

FIG. 5 (a) Eye diagram of the decoded incoming $2^{7}-1$ PRBS OCDMA data by the receiver (b) Example of the decoded incoming OCDMA data representing "1010" data stream (c) Example of all-optically recovered clock from a received OCDMA data by AOCR.

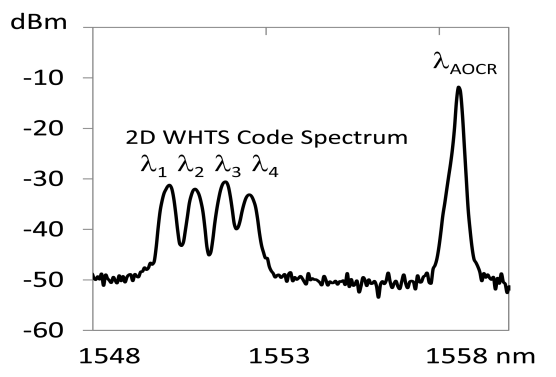

FIG. 6 Recorded optical spectrum of 2D-WHTS OCDMA code; $\lambda$ AOCR belongs to the all-optically recovered clock signal.

head (Agilent 86105B) were used for the monitoring. Then measured BERs were plotted and eye diagrams recorded.

Figure 5(a) and 5(b) shows obtained eye diagrams of the decoded 27-1 PRBS OCDMA data and 1010 decoded data pattern, respectively. Figure 5 (c) is an all-optically recovered clock as seen by a bandwidth limited oscilloscope with $20 \mathrm{GHz}$ sampling head.

For the illustration, Figure 6 shows the combine output spectrum from the AOCR and OCDMA decoder recorded by an optical spectrum analyser. The signal on the left is the spectrum of the 2D-WHTS OCDMA codes (four wavelengths $\lambda_{1}-\lambda_{4}$ could be noted). The peak on the right is the all-optically recovered clock signal at the wavelength $\lambda_{A O C R}=1558 \mathrm{~nm}$ ) generated by the AOCR from the received OCDMA data.

For the illustration, Figure 7 shows two obtained BER results. Figure $7(\mathrm{a})$ was obtained when the optical clock recovered from the received $2^{7}-1$ PRBS data was used for synchronization. Figure 7(b) was obtained when we used RF synthesizer for clock generation. As can be seen, approximately $7.5 \mathrm{~dB}$ power budget improvement was noted when using the all optically recovered clock. No error floor was observed in both cases. 


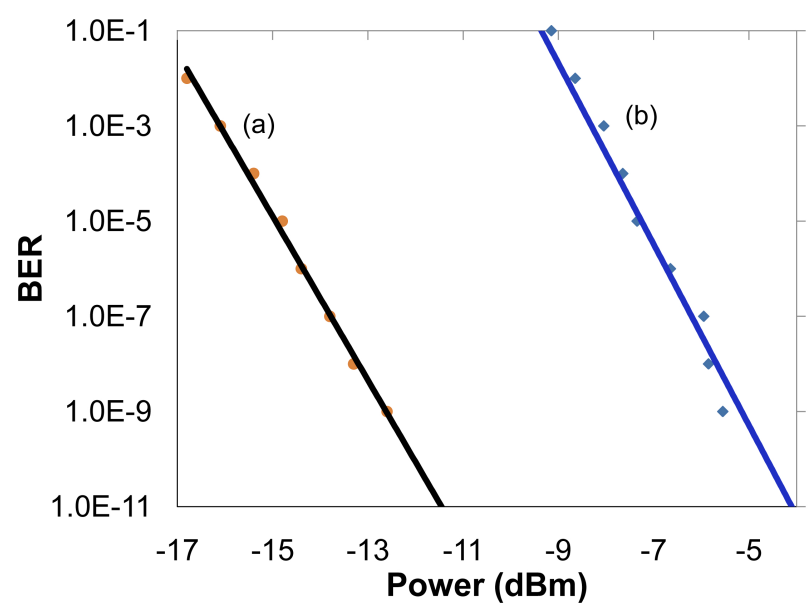

FIG. 7 Measured BER for decoded $2^{7}-1$ PRBS data pattern when:

(a) Synchronization was done by using all-optically recovered clock from received OCDMA data

(b) Synchronization is done directly by the clock generated from the RF synthesiser.

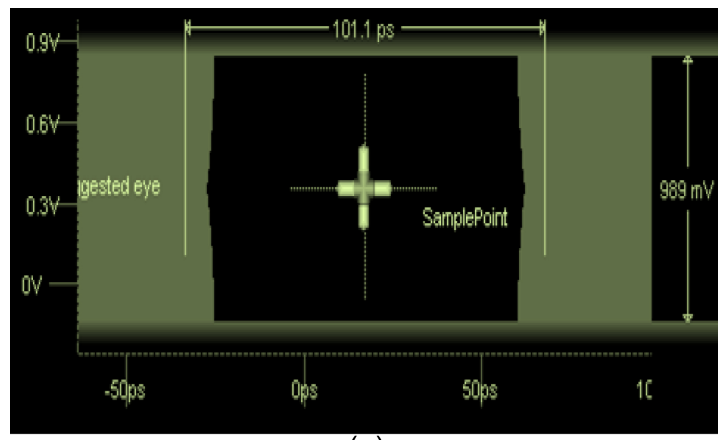

(a)

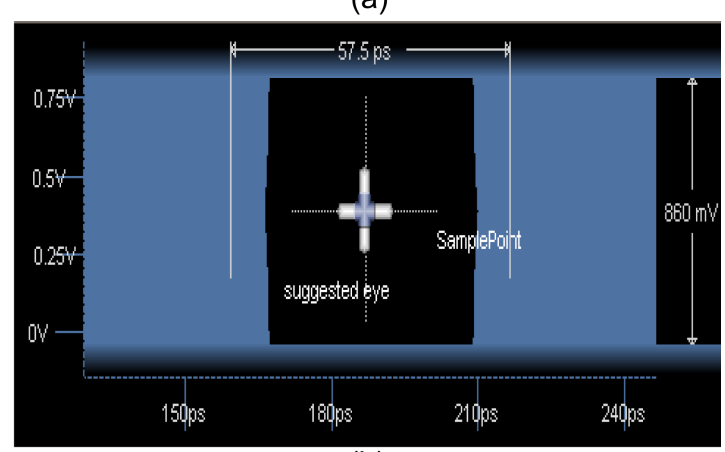

(b)

FIG. 8 Obtained eye diagrams as recorded by BER tester (Agilent N4903A) for error free system operation when:

(a) All-optically recovered clock was used for synchronization

(b) Direct clock from the RF synthesizer was used for the synchronization.

Using the all-optically recovered clock for the synchronization also resulted in a wider and bigger eye opening: 101.1 ps width and $989 \mathrm{mV}$ height if compare to $57.5 \mathrm{ps}$ width and $860 \mathrm{mV}$ height when using the local clock from the RF synthesizer. This can be seen in the Figure 8(a) and 8(b) as recorded by the bit error rate tester (Agilent N4903A). These results are complimentary to Figure $7(\mathrm{a})$ and $7(\mathrm{~b})$, respectively when the system was running error free. These improvements can be explained by the fact that the all-optically recovered clock when used to synchronize the receiver helps the receiver to "track" the OCDMA signal under the influence of timing jitter that may arise due to transmit power variation, group velocity residual dispersion or thermal coefficients of the transmission

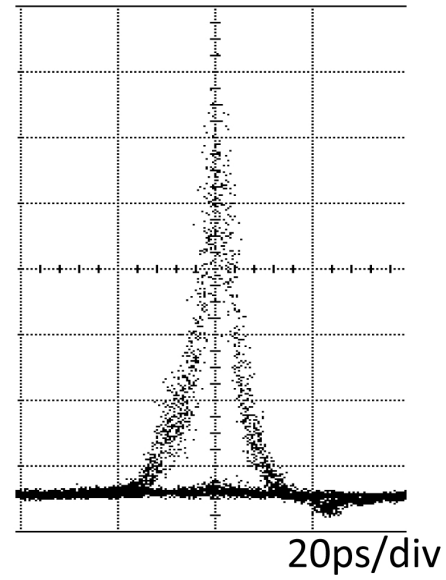

FIG. 9 Eye diagram recorded from oscilloscope for the application of recovered clock to drive $2 \times 2$ SOA-based MZI switch.

link. This tracking is therefore helping to "eliminate" the effect of timing jitter and improves BER [12].

In order to test the clock recovered all-optically from the received OCDMA signal for the "ability" to drive the picosecond SOA-MZIS, (TG) a second experiment was conducted. Here, the all-optically recovered clock signal by AOCR was used as a control signal for the TG as shown in detail in Figure 1 and 2. First, 8 ps switching window to match the autocorrelation width was set by adjusting the optical delay line (ODL2). At the switch output low pass filter, F blocks the TG's control signal and passes the autocorrelation (at this point already stripped of the MAI by the TG). The experimental results showing the autocorrelation after the time filtering by the TG can be seen in Figure 9 as recorded by the bandwidth limited oscilloscope with a $20 \mathrm{GHz}$ optical sampling head, (Agilent 86105B).

\section{CONCLUSION}

We have developed and successfully demonstrated an incoherent OCDMA receiver with built-in all-optical clock recovery. The recovered optical clock was then successfully used for the self-synchronization of the picosecond SOA-based MachZehnder interferometric all-optical switch as the time gate for the MAI suppression.

\section{ACKNOWLEDGEMENT}

Siti Idris is on leave from Universiti Teknikal Malaysia Melaka (UTeM) and her PhD work is supported by the Ministry of Higher Education Malaysia.

\section{References}

[1] L. Tancevski, and I. Andonovic, "Wavelength Hopping Time Spreading Code Division Multiple Access Systems," Electron. Lett. 30, 1388-1390 (1994).

[2] P. R. Prucnal, Optical Code Division Multiple Access: Fundamental and Applications (Taylor and Francis, Boca Raton, 2004). 
[3] Y.-K. Huang, V. Baby, I. Glesk, C.-S. Brès, C. M. Greiner, D. lazikov, T. W. Mossberg, and P. R. Prucnal, "Novel multicode-processing platform for wavelength-hopping time-spreading optical CDMA: A path to device miniaturization and enhanced network functionality," IEEE J. Sel. Top. Quantum Electron. 13, 1471-1479 (2007).

[4] V. Jyoti, and R. S. Kaler, "Design and implementation of 2-dimensional wavelength/time codes for OCDMA," Int. J. Light Electron Opt. 122, 851-857 (2010).

[5] V. Baby, C.-S. Brès, L. Xu, I. Glesk, and P. R. Prucnal, “Demonstration of differentiated service provisioning with 4-node $253 \mathrm{Gchip} / \mathrm{s}$ fast frequency hopping time spreading OCDMA," Electron. Lett. 40, 755-756 (2006).

[6] N. T. Dang, A. T. Pham, and Z. Cheng, "Impact of GVD on the performance of 2-D WH/TS OCDMA system using heterodyne detection receiver," IEICE Trans. Fund. E 92-A, 1182-1191 (2009).

[7] L. Tancevski, and L. A. Rusch, "Impact of the beat noise on the performance of 2D optical CDMA System," IEEE Commun. Lett. 4, 264-266 (2000).

[8] T. V. Lerber, S. Honkanen, A. Tervonen, H. Ludvigsen, and F. Kuppers, "Optical clock recovery method: review," Elsevier Opt. Fiber Technol. 15, 363-372 (2009).

[9] K. Vlachos, G. Theophilopoulos, A. Hatziefremidis, and H. Avramopoulos, "30 Gb/s all optical clock recovery circuit," IEEE Photon. Technol. Lett. 12, 705-707 (2000).

[10] Y. Su, L. Wang, A. Agrawal, and P. Kumar, "Wavelength-tunable all-optical clock recovery using a fiber-optic parametric oscillator," Opt. Commun. 184, 151-156 (2000).

[11] L. F. K. Lui, A. Zhang, P. K. A. Wai, H. Y. Tam, and M. S. Demokan, " $40 \mathrm{~Gb} / \mathrm{s}$ All-optical clock recovery based on an optical parametric oscillator with photonic crystal fiber," in Proceedings to Lasers and Electro-Optics, 2008 and 2008 Conference on Quantum Electronics and Laser Science, 1-2 (CLEO/QELS, California, 2008).

[12] S. Zhang, F. Comez-Agis, Y. Liu, N. Calabretta, E. Tangdiongga, and H. J. S. Dorren, "Fast-synchronization and low-timing jitter selfclocking concept for $160 \mathrm{Gbit} / \mathrm{s}$ optical time-division multiplexing transmissions," Opt. Lett. 35, 37-39 (2010).

[13] Y. Deng, K. Kravtsov, M. P. Fok, and P. R. Prucnal, K. Sasaki, C. C. Gupta, and S. Kobayashi, "All-optical asynchronous detection for a compact integrable incoherent optical CDMA system," J. Light. Technol. 27, 5370-5375 (2009).

[14] J. Faucher, S. Ayotte, L. A. Rusch, S. LaRochelle, and D. Plant, "Experimental BER performance of 2-D-t OCDMA with recovered clock," Electron. Lett. 41, 713-715 (2005).

[15] K. Kravtsov, Y. Deng, and P. R. Prucnal, "Self-clocked all- optical add/drop multiplexer for asynchronous CDMA ring networks," IEEE J. Quantum Electron. 45, 396-401 (2009).

[16] J. P. Sokoloff, P. R. Prucnal, I. Glesk, and M. Kane, "A terahertz optical asymmetric demultiplexer (TOAD)," IEEE Photon. Technol. Lett. 5, 787-790 (1993).

[17] G.-C. Yang, and W. C. Kwong, Prime Codes with Applications to CDMA Optical and Wireless Networks (Artech House, Norwood, 2002).
[18] C.-S. Brès, V. Baby, I. Glesk, L. Xu, D. Rand, and P. R. Prucnal, "Scalability of Frequency- Hopping Time Spreading OCDMA Code Matrix," in Proceedings to Conference on Laser and Electro-Optics 2004, (CLE0, California, 2004).

[19] I. Glesk, V. Baby, C.-S. Brès, Y.-K. Huang, and P. R. Prucnal, "Performance enhancement of optical CDMA systems using ultrafast all-optical sampling," in Proceedings to IEEE Conference on Avionics Fiber-Optics and Photonics, 2000, 58-59 (AVFOP, Minneapolis, 2005).

[20] Y.-K. Huang, K. Kravtsov, I. Glesk, P. R. Prucnal, C. M. Greiner, D. lazikov, and T. W. Mossberg, "Integration of dual-code optical CDMA encoder and decoder by holographic Bragg reflectors," in Proceedings to Optical Fiber Communication and the National Fiber Optic Engineers Conference, 2007, 1-3 (OFC/NFOEC, Anaheim, 2007).

[21] Y. Zhang, H. Chen, Z. Si, H. Ji, and S. Xie, "Design of FBG en/decoders in coherent 2-D time-wavelength OCDMA systems," IEEE Photon. Technol. Lett. 20, 891-893 (2008).

[22] T. B. Osadola, S. K. Idris, I. Glesk, K. Sasaki, G. C. Gupta, "In Situ Method for Power Re-Equalization of Wavelength Pulses Inside of OCDMA Codes," IEEE J. Quantum Electron. 47, 1053-1058 (2011).

[23] I. Andonovic, L. Bazgaloski, M. Shabeer, and L. Tancevski, “Incoherent all optical code recognition with balanced detection," J. Light. Technol. 12, 1073-1080 (1994).

[24] I. Glesk, P. R. Prucnal, and I. Andonovic, "Incoherent Ultrafast OCDMA Receiver Design with 2 ps All-optical Time Gate to Suppress Multiple-Access Interference," IEEE J. Sel. Top. Quantum Electron. 14, 861-867 (2008).

[25] S. Nakamura, Y. Ueno, and K. Tajima, "Ultrahigh-speed optical signal processing with symmetric-Mach-Zehnder-type alloptical switches," in Proceedings of All-optical Networking: Existing and Emerging Architecture and Applications/Dynamic Enablers of Next-Generation Optical Communications Systems/Fast Optical Processing in Optical Transmission/VCSEL 2002, TuK4-27-TuK-28 (IEEE/LEOSST, Quebec, 2002).

[26] P. Bakopoulos, D. Tsiokos, 0. Zouraraki, H. Avramopoulos, G. Maxwell, and A. Poustie, "Compact all-optical packet clock and data recovery circuit using generic integrated MZI switches," Opt. Express 13, 6401-6406 (2005).

[27] I. Glesk, P. Bock, P. Cheben, J. Schmid, J. Lapointe, and S. Janz, "Alloptical switching using nonlinear subwavelength Mach-Zehnder on silicon," Opt. Express 19, 14031-14039 (2011).

[28] H. L. Minh, Z. Gbassemlooy, and W. P. Ng, "Ultrafast All- optical Self Clock Extraction Based on Two Inline Symmetric Mach-Zehnder Switches," in Proceedings of International Conference of Transparent Optical Networks 2006, 64-67 (ICTON, Nottingham, 2006).

[29] S. Idris, T. Osadola, and I. Glesk, "OCDMA receiver with built-in alloptical clock recovery," in Proceedings of European Optical Society Annual Meeting 2012, (EOSAM, Aberdeen, 2012). 